\title{
An equivalent single-phase flow for oil-water two-phase flow and its potential application in well test
}

\author{
Wenshu Zha ${ }^{1}$, Daolun $\mathrm{Li}^{1}{ }^{*}$, Zhiwei $\mathrm{Lu}^{2}$, Bao $\mathrm{Jia}^{3}$ \\ ${ }^{1}$ School of Mathematics, Hefei University of Technology, Hefei 230009, P. R. China \\ ${ }^{2}$ Vertibi School of Engineering, University of Southern California, Los Angeles, California 90089, USA \\ ${ }^{3}$ Chemical \& Petroleum Department, University of Kansas, Lawrence, Kansas 66045, USA
}

(Received April 16, 2018; revised May 4, 2018; accepted May 5, 2018; available online May 9, 2018)

\section{Citation:}

Zha, W., Li, D., Lu, Z., Jia, B. An equivalent single-phase flow for oil-water two-phase flow and its potential application in well test. Advances in Geo-Energy Research, 2018, 2(2):

218-227, doi: 10.26804/ager.2018.02.09.

Corresponding author:

*E-mail: 1daol@hfut.edu.cn

Keywords:

Equivalent single-phase flow

equivalent viscosity

two-phase flow

numerical well test

PEBI grid

\begin{abstract}
:
In this work an equivalent single-phase flow model is proposed based on the oil-water twophase flow equation with saturation-dependent parameters such as equivalent viscosity and equivalent formation volume factor. The equivalent viscosity is calculated from the oilwater relative permeability curves and oil-water viscosity. The equivalent formation volume factor is obtained by the fractional flow of the water phase. In the equivalent single-phase flow model, the equivalent viscosity and phase saturation are interdependent when the relative permeability curves are known. Four numerical experiments based on PEBI grids show that equivalent single-phase flow has a good agreement with the oil-water two-phase flow, which shows that the equivalent single-phase flow model can be used to interpret oil-water two-phase pressure data measured in the wellbore during the buildup period. Because numerical solution of single-phase flow model is several times faster than that of the two-phase flow model, whether the new model interprets the pressure data directly or offers good initial values for the true oil-water two-phase pressure data interpretation, it will obviously improve the efficiency of the interpretation of oil-water pressure data and decrease the burden of engineers.
\end{abstract}

\section{Introduction}

Reliable description of petroleum reservoirs is essential for accurate performance forecasting. Besides geology and seismology, several branches of the petroleum industry have contributed in describing petroleum reservoirs, for example, well testing, and well logging. Well testing is an efficient tool capable of estimating in-situ reservoir properties and has been used for many years to evaluate well and reservoir parameters like permeability, skin, average pressure, etc. Well testing has been increasingly important in reservoir management, due to the importance of reservoir and well parameters it offers.

Multiphase flow is commonly encountered in reservoirs. Equations describing multiphase flow are ususally highly nonlinear that analytical solutions are difficult to be obtained. Previously a lot of work has been performed to incorporate multiphase flow effects into single-phase flow theory. Methodogies adopted by previous publications can be divided into two main categories. The first one is the pressure (Perrine's) approach. The second one is the pseudo pressure (Raghavan's) approach. Penine (1956) provided a modified single-phase flow theory to incorporate the multi-phase flow effects. He replaced single-phase mobility term, $k / \mu$, by the total mobility $\sum_{i=o, g, w}(k / \mu)_{l}$, and replaced single-phase compressibility with the total-system compressibility. Earlougher et al (1977), Fetkovich and Vienot (1984), Raghavan (1986), Chu et al. (1986), and Ayan and Lee (1986) further studied Pemne's approach. Raghavan (1976) utilized pseudo pressure approach to analyze the buildup and drawdown tests in solution gasdrive reservoirs to estimate absolute permeability and wellbore skin phenomenon. In recent years, Thompson and Reynolds (1997) and Raghavan (2009) also studied the multiphase pressure analysis.

The previous approaches involved too much mathematical simplifications during the derivation of the single-phase equation. In additional, the above approaches did not interprete the saturation distribution based on the single-phase flow equation from the multiphase flow, because of the limitation of the analytical solution.

Although the analytical method is fast and reliable, it is developed specially for idealized reservoir configurations and 
only suitable for simple cases. Therefore, the numerical well test approach has developed. For instance, the perpendicular bisection (PEBI) grid (Palagi and Azziz, 1991) has been widely used in commercial well test software (Nnadiand Onyekonwu, 2004; Alcalde and Teufel, 2006; Pinzon et al., 2010).

Numerical well test technique simulating the multiphase flow has been widely used in the oil field. However, there are two main challenges while interpreting the multiphase pressure data measured in the wellbore during buildup period. The first is that the grids around the wells should be small enough to simulate the transient pressure, leading to the very small timestep. In addition, during numerical well test interpretation, the boundary condition is difficult to decide which is greatly different from numerical reservoir simulation. The common practice to deal with boundary condition is to add many wells around the measured well, resulting in the second challenge that too many grids are present. In a short summary, in the process of interpreting the multiphase flow pressure data, the small grid and the large number of grids make the pressure data interpretation a time-consuming process.

The economic production of unconventional reservoir largely depends on the dense and conductive fracture network generated during the hydraulic fracturing process in the well drainage volume, known as the stimulated reservoir volume (SRV), which adds the complexity of numerical well tests (Li et al., 2014; Zhao et al., 2016; Cai et al., 2017; Zhang et al., 2017). The recent research shows that the pseudo TPG (threshold pressure gradient) is the the reason of the positive pressure derivative for wells in ultra-low permeability reservoirs ( $\mathrm{Li}$ et al., 2016). Besides, the non-Darcy flow phenomenon adds the complexity of numerical well test in unconventional reservoirs.

In this work, we aimed to decrease the interpretation speed, apply different techniques to reduce oil-water two-phase flow equations to equivalent single-phase flow (ESPF) equation using pressure and saturation dependent paramters. The only simplification in our approach is that the saturation distribution is stable and remains the same during the numerical simulation. Our results show that during a short time span, the ESPF model has a good agreement in comparison to the true oilwater two-phase model, which shows that the ESPF model can be used to interpret multiphase pressure data.

\section{Methodology}

\subsection{Mathematics model}

The two-phase flow equations are expressed as

$$
\nabla \bullet\left[\frac{K K_{r o}}{\mu_{o} B_{o}}\left(\nabla P_{o}-\gamma_{o} \nabla Z\right)\right]=\frac{\partial}{\partial t}\left(\frac{\phi S_{o}}{B_{o}}\right)-q_{o s c}
$$

and

$$
\nabla \bullet\left[\frac{K K_{r w}}{\mu_{w} B_{w}}\left(\nabla P_{w}-\gamma_{w} \nabla Z\right)\right]=\frac{\partial}{\partial t}\left(\frac{\phi S_{w}}{B_{w}}\right)-q_{w s c}
$$

The oil rate production rate from a well is expressed as

$$
q_{o s c}=\frac{2 \pi K K_{r o} h}{\mu_{o} B_{o}\left[\ln \left(r_{e} / r_{w}\right)+S\right]}\left(p_{i}-p_{w f}\right)
$$

The water rate production rate from a well is expressed as

$$
q_{w s c}=\frac{2 \pi K K_{r w} h}{\mu_{w} B_{w}\left[\ln \left(r_{e} / r_{w}\right)+S\right]}\left(p_{i}-p_{w f}\right)
$$

Ingoring gravity and capillary effects, Eqs. (3) and (4) become

$\nabla \bullet\left[\left(\frac{K K_{r o}}{\mu_{o} B_{o}}+\frac{K K_{r w}}{\mu_{w} B_{w}}\right) \nabla p\right]=\frac{\partial}{\partial t}\left[\phi\left(\frac{S_{o}}{B_{o}}+\frac{S_{w}}{B_{w}}\right)\right]-\left(q_{o s c}+q_{w s c}\right)$

The fractional flow of the water phase is reagrranged as

$$
\alpha=\frac{\frac{K_{r w}}{\mu_{w}}}{\frac{K_{r o}}{\mu_{o}}+\frac{K_{r w}}{\mu_{w}}}
$$

We have

$$
\begin{aligned}
& \frac{K K_{r o}}{\mu_{o} B_{o}}+\frac{K K_{r w}}{\mu_{w} B_{w}} \\
& =\frac{K}{B_{o}}(1-\alpha)\left(\frac{K_{r o}}{\mu_{o}}+\frac{K_{r w}}{\mu_{w}}\right)+\frac{K}{B_{w}} \alpha\left(\frac{K_{r o}}{\mu_{o}}+\frac{K_{r w}}{\mu_{w}}\right) \\
& =K\left(\frac{(1-\alpha)}{B_{o}}+\frac{\alpha}{B_{w}}\right)\left(\frac{K_{r o}}{\mu_{o}}+\frac{K_{r w}}{\mu_{w}}\right)
\end{aligned}
$$

Let

$$
\begin{gathered}
\frac{1}{B_{e}}=\frac{(1-\alpha)}{B_{o}}+\frac{\alpha}{B_{w}} \\
\frac{1}{\mu_{e}}=\frac{K_{r w}}{\mu_{w}}+\frac{K_{r o}}{\mu_{o}} \\
\frac{1}{B_{a}}=\frac{S_{o}}{B_{o}}+\frac{S_{w}}{B_{w}}
\end{gathered}
$$

where, $\mu_{e}$ is the equivalent viscosity, and $B_{e}$ is the equivalent formation volume factors, and $B_{a}$ is the equivalent accumulation formation volume factor.

Based on Eqs. (8), (9) and (10), Eq. (5) can be reduced to:

$$
\nabla \bullet\left[\frac{K}{\mu_{e} B_{e}} \nabla p\right]=\frac{\partial}{\partial t}\left[\frac{\phi}{B_{a}}\right]-q_{e s c}
$$

From the Eqs. (8) and (9), the total liquid production can be written as:

$$
q_{e s c}=q_{o s c}+q_{w s c}=\frac{2 \pi K h}{\mu_{e} B_{e}\left[\ln \left(r_{e} / r_{w}\right)+S\right]}\left(p_{i}-p_{w f}\right)
$$

If wellbore storage effect is considered, Eq. (12) can be written as:

$$
q_{e s c}=\frac{2 \pi K h}{\mu_{e} B_{e}\left[\ln \left(r_{e} / r_{w}\right)+S\right]}\left(p_{i}-p_{w f}\right)-C \frac{\partial p_{w f}}{\partial t}
$$

In this work, the model described by Eqs. (11) and (13) is named as equivalent single-phase flow model. 


\subsection{Equation discretization}

The ESPF equation is nonlinear and has analytical solution in geologically simple reservoir. However, due to the limitation of the analytical solution, numerical solution method is adopted here based on the PEBI grids. Using the control volume methodthe discretized form of the flow Eq. (11) of fully implicit time is

$$
\begin{aligned}
& \sum_{j} T_{i j, e}^{n+1}\left[p_{j}-p_{i}\right]^{n+1} \\
& =\frac{V_{i}}{\triangle t}\left[\frac{\phi^{r e f} C_{r}}{B_{a}^{n}}+\phi^{n+1}\left(\frac{S_{o} C_{o}}{B_{o}^{r e f}}+\frac{S_{w} C_{w}}{B_{w}^{r e f}}\right)\right]\left(p_{i}^{n+1}-p_{i}^{n}\right) \\
& -Q_{e s c}^{n+1}
\end{aligned}
$$

where $T_{i j, e}$ is the transmissibility between a pair of blocks $i$ and $j$, the oil and water formation volume factors and porosity are approximated by the following equation respectively:

$$
\begin{gathered}
B_{l}=\frac{B_{l}^{r e f}}{1+C_{l}\left(p-p^{r e f}\right)}, l=o, w \\
\phi=\phi^{r e f}\left[1+C_{r}\left(p-p^{r e f}\right)\right]
\end{gathered}
$$

The assumption in the equivalent single-phase flow model is that phase saturation is a constant value and does not change. Therefore, Eq. (14) is single phase flow, and the $\mu_{e}, B_{e}$ and $B_{a}$ first decided by initial saturation and pressure, and change with pressure during simulating.

For the oil-water two-phase model, the single point upstream weighting scheme and linearly implicit time stepping methods are adopted, and Newton iteration method is used to solve the nonlinear equation.

\subsection{Two-phase flow converting to equivalent single phase flow}

At beginning of the simulation, the pressure and phase saturation distribution are given. The relative permeability $K_{r o}$, $K_{r w}$, phase viscosities $\mu_{w}, \mu_{o}$ and formation volume factors $B_{o}, B_{w}$ can be calculated with the given pressure and phase saturation.

With these known basic parameters $K_{r o}, K_{r w}, \mu_{w}, \mu_{o}, B_{o}$ and $B_{w}$, the equivalent viscosity $\mu_{e}$ and equivalent formation volume factors $B_{a}$ can be obtained from Eqs. (9) and (10) respectively. The fractional flow of the water phase $\alpha$ can be obtained from Eq. (6), and then the equivalent formation volume factors $B_{e}$ can be calculated from Eq. (8). With the parameters such as $\mu_{e}, B_{a}, B_{e}$, the pressure distribution can be solved by ESPF model described by Eqs. (11) and (13).

\subsection{Get saturation from the equivalent viscosity}

In the equivalent single-phase flow, the equivalent viscosity $\mu_{e}$ and the phase saturation $S_{w}$ or $S_{o}$ are interdependent. The viscosity $\mu_{e}$ can be obtained from Eq. (9). At the same time, when the equivalent viscosity $\mu_{e}$ is known, Eq. (9) can interpret the phase saturation with the known basic parameters such as the oil and water viscosities, relative permeability curves. The algorithm to obtain the saturation from the equivalent viscosity $\mu_{e}$ is given by the following process.

The algorithm 1: Get saturation from the equivalent viscosity $\mu_{e}$.

1. Input oil and water relative permeability curves, viscosities curves or correlations of oil and water, pressure $P$, increase of saturation step $\Delta S$, and equivalent viscosity $\mu_{e}$;

2. First, let $S_{w}$ be the minimum water saturation;

3. Use linear interpolation to estimate the relative permeability $K_{r w}$ and $K_{r o}$ at the water saturation $S_{w}$ from the given relative permeability curves;

4. Calculate the value of $\mu_{o}, \mu_{w}$ at pressure $P$ from the given viscosities curves or correlations;

5. If the Eq. (9) is not satisfied, make the oil saturation $S_{o}=S_{w}+\Delta S$ and go to the step (3) and continue;

6. If the Eq. (9) is satisfied, the water saturation $S_{w}$ is obtained and oil saturation $S_{o}=1-S_{w}$, stop.

The ESPF model assumes that the oil saturation $S_{o}$ is stationary. The equivalent viscosity $\mu_{e}$ at the beginning of the simulation depends on initial pressure and initial oil saturation $S_{o}$ and is noted as $\mu_{e}^{r e f}$. The value $\mu_{e}$ varies in time and can be written as $\mu_{e}=\mu_{e}^{r e f}$. It is $\mu_{e}^{r e f}$ that used to interpret the saturation but not the changing value $\mu_{e}$.

\section{Usage of the equivalent single-phase flow model}

The assumption of the method is that the saturation is stationary and does not change with time, which limits its application.

Normally, well tests span a short production time, during which the reservoir saturation distribution changes a little and can be approximately assumed constant. Therefore, the ESPF model is suitable for well test analysis.

The main usage of the ESPF model is the well test. There are two ways to use it. ESPF is used to interpret measured pressure data directly or is used to give a good initial estimation for oil-water two-phase pressure data, which will improve the speed of pressure data interpretation.

\subsection{The pressure data interpretation with known ab- solute permeability}

In the old fields, for example in China old fields, there are so many wells that reservoir parameters like permeability are well known. The reservoir engineers are eager to know where the residual oil is by an economic way. The ESPF offers a quick and economic method to interpret the saturation distribution based on the measured pressure.

The characteristic of this method is that given the distribution of the absolute permeability, the equivalent viscosity $\mu_{e}$ and other parameters can be estimated by matching the history pressure and pressure derivative. After the equivalent viscosity $\mu_{e}$ is obtained, oil and water saturation will be interpreted according to the algorithm 1 . The algorithm 2 below gives the details. 


\section{Algorithm 2: Get saturation distribution from the pressure measured in the shut-in well using the ESPF model.}

1. Input basic parameters such as relative permeability curves, viscosities and formation volume factors of oil and water, the initial pressure $p$, absolute permeability distribution;

2. Give an initial guess for the equivalent viscosity distribution $\mu_{e, i}, i=1,2, \ldots, n$;

3. Give an initial guess for well storage, skin and other parameters;

4. Use the algorithm 1 to obtain the phase saturation $S_{w, i}$ and $S_{o, i}$ from the initial guess $\mu_{e, i}$;

5. Calculate equivalent accumulation formation volume factors $B_{a}$ given by Eq. (10) based on the saturation $S_{w, i}$ and $S_{o, i}$ obtained in step 4 and the formation volume factors given in step (1);

6. Calculate the equivalent formation volume factor $B_{e}$ by Eq. (8) based on the saturation $S_{w, i}$ calculated in step 4 and other parameters given in step 1 ;

7. Perform simulation based on the ESPF model by the numerical way and obtain the theoretical value of pressure and pressure derivative;

8. If the theoretical value of pressure fits the measured value of pressure, the guess is correct and the saturation distribution obtained based on the guess is correct too;

9. If the theoretical value of pressure does not fit the measured value of pressure, change the value of initial guess and return step 4 .

\subsection{Pressure data interpretation without known abso- lute permeability}

The characteristic of this method is that the absolute permeability, equivalent viscosity $\mu_{e}$ and other parameters are needed to be estimated by matching the pressure history and pressure derivative. Therefore, this method is similar to algorithm 2 and the procedures are not repeted here.

In the equivalent single-phase flow model, the equivalent viscosity $\mu_{e}$ determines the value of water and oil saturation and indirectly decides the equivalent accumulation formation volume factor $B_{a}$. Therefore, the equivalent single-phase flow can distinguish the influence of the viscosity and absolute permeability, and that the equivalent viscosity and absolute permeability are the parameters to be matched is reasonable and practical.

\section{Numerical simulation results}

In this section, numerical experiments are conducted to investigate the effectiveness of the method. The effectiveness of the ESPF model needs to be evaluated which is the core of this study. The only assumption of the ESPF model is that the saturation distribution is stationary, while for the true oilwater two-phase flow, saturation distribution varies with time. Therefore, the examples below aimed to show that assumption of stationary saturation only lead to very small errors in a short time span.
Four numerical examples of equivalent single-phase flow are illustrated to compare to that of oil-water two-phase flow. (1) Reservoir is homogeneous, and saturation distribution is uniform. Formation volume factors for the oil and water are equal to each other. Under these conditions, the simulation results are free from the influence of formation volume factors so as to verify the effectiveness of the method; (2) The oil saturation distribution is not uniform, and formation volume factors of oil and water are not equal to each other; (3) In the previous two experiments, the closed boundary conditions are adopted. However, in this experiment, the constant pressure boundary conditions are adopted, to see the performance of ESPF model under different boundary conditions; (4) one production well, four injection wells are present. Two different saturation distributions are given to show the performance of the ESPF model. One large saturation difference is 0.3 , and one small difference of 0.1 are applied that the latter one is more realistic.

The reservoir has an area of $400 \mathrm{~m} \times 400 \mathrm{~m}$ and the grid is shown as Fig. 2(a). The absolute permeability in the first numerical experiment is $101 \mathrm{mD}$, while the absolute permeability in other 3 experiments is $700 \mathrm{mD}$. Reference formation volume factors $B_{o}^{r e f}$ and $B_{w}^{r e f}$ are 1.05 in the first numerical experiment, while in other 3 experiments the $B_{o}^{r e f}$ is 1.2 , and $B_{w}^{r e f}$ is 1 . The relative permeability of oil and water is given in Table 1. In all the experiments, only experiment 3 is applied with constant pressure boundary conditions, whereas others are applied with closed boundary conditions.

The liquid is produced at constant rate $80 \mathrm{~m}^{3} /$ day in the production well for 20 days, and water is injected at a constant rate of $20 \mathrm{~m}^{3} /$ day in the injection well at the surface condition for 30 days. Then the production well shuts for 10 days. The wellbore storage is $1 \mathrm{~m}^{3} /$ day, and the wellbore skin is zero. The Peaceman type well model is used.

In Table 1, connate water and residual oil saturation are assumed to be zero for simplicity, note that. in real oil reservoir connate water and residual oil saturation are always larger than zero. According to the relative permeability in Table 1, the equivalent viscosity for oil saturation of 0.4 is $7.1712 \mathrm{mPa} \cdot \mathrm{S}$, and for oil saturation of 0.7 the value is $17.6 \mathrm{mPa} \cdot \mathrm{S}$.

\subsection{Experiment 1: A uniform saturation distribution with equal formation volume factors}

The reservoir is assumed to be homogeneous and the saturation distribution is uniform. The formation volume factors for the oil and water are the same. Oil-water two-phase flow model and the ESPF model are used to simulate the flow and see their difference. Fig. 1 gives the curve of oil-water twophase flow marked by square and the curve of ESPF marked by up triangle.

In Fig. 1(a), we can see that only a very small difference exists in pressure drawdown period between the equivalent single phase and the oil-water two-phase and almost no difference exists in pressure buildup period. Fig. 1(b) shows that there is almost no difference in the log-log figure between the equivalent single phase and the oil-water two-phase. This 
Table 1. Relative permeability.

\begin{tabular}{lll}
\hline$S_{w}$ & $K_{r w}$ & $K_{r o}$ \\
\hline 0 & 0 & 1 \\
0.1 & $1.00 \times 10^{-5}$ & 0.729 \\
0.2 & 0.00032 & 0.512 \\
0.3 & 0.00243 & 0.343 \\
0.4 & 0.01024 & 0.216 \\
0.5 & 0.03125 & 0.125 \\
0.6 & 0.07776 & 0.064 \\
0.7 & 0.16807 & 0.027 \\
0.8 & 0.32768 & 0.008 \\
0.9 & 0.59049 & 0.001 \\
1 & 1 & 0 \\
\hline
\end{tabular}

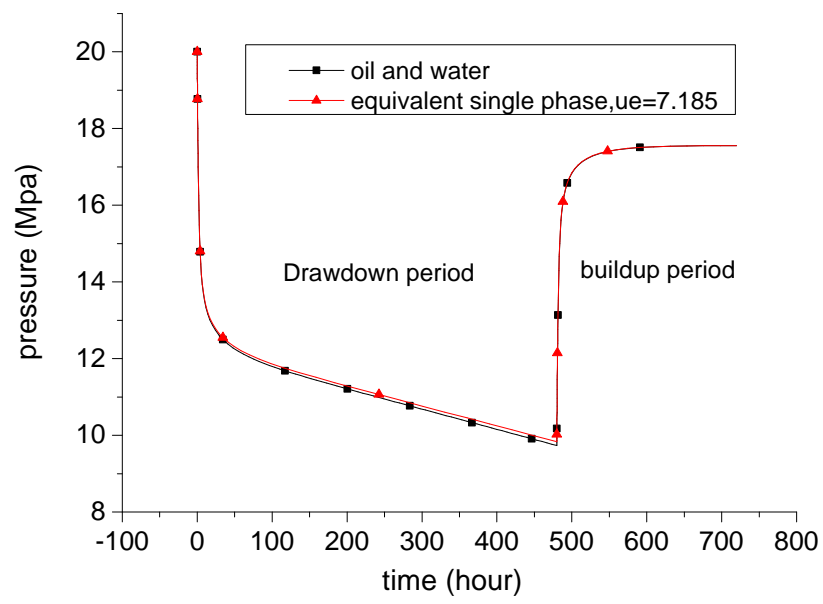

(a) Bottomhole pressure of the drawdown and buildup period

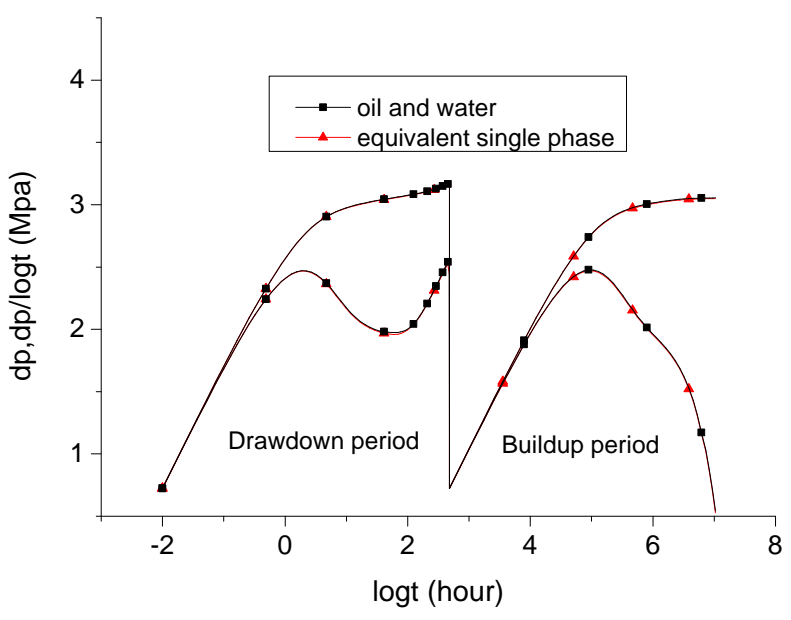

(b) Pressure drop and pressure derivative

Fig. 1. Comparison of two-phase flow and ESPF with $B_{w}^{r e f}=B_{o}^{r e f}$.

is very important because the pressure buildup period is what we are concerned in the well test.

\subsection{Experiment 2: Saturation distribution and differ- ent formation volume factors}

In this experiment, oil saturation distribution is heterogeneous. The oil saturation in one region is 0.7 and 0.4 in the other region as shown in Fig. 2(a).

Fig. 2(b) gives the log-log curves of true oil-water twophase flow with uneven saturation distribution marked by square, and curves of the corresponding ESPF marked by diamond. The curve of true oil-water two-phase flow with uniform saturation distribution is also given and used to see whether ESPF can lead to misleading interpretation, which marked by up triangle.

Fig. 2(b) shows that the ESPF also has a very good agreement with the two-phase flow, which means that the ESPF is effective when saturation distribution is not uniform. Fig. 2(b) also shows that the curve of oil-water two-phase with a uniform saturation distribution is separate from the curve of ESPF with an uneven saturation distribution. One might argue that the two curves are only detached at the late period, which will influence the interpretation effects. Our explanation is that the region with oil saturation of 0.7 is small and far away from the well.

In order to see the influence of the distance of the region with different oil saturation, another example is given in Fig. 3.

The region with oil saturation of 0.7 in Fig. 3(a) is much bigger and nearer to the well compared to Fig. 2(a). Fig. 3(b) shows that the ESPF and two-phase flow also has a good agreement. We should notice the fact from Fig. 3(b) that although the equivalent single-phase and two-phase flow has a good agreement in log-log curves, the difference between them is bigger than the previous experiments. The reason is that the region with $S_{o}=0.7$ is close to the well, and the saturation boundary is very sharp, which leads to a much bigger saturation change in the actual reservoir while in the equivalent single-phase model the oil saturation is a constant 


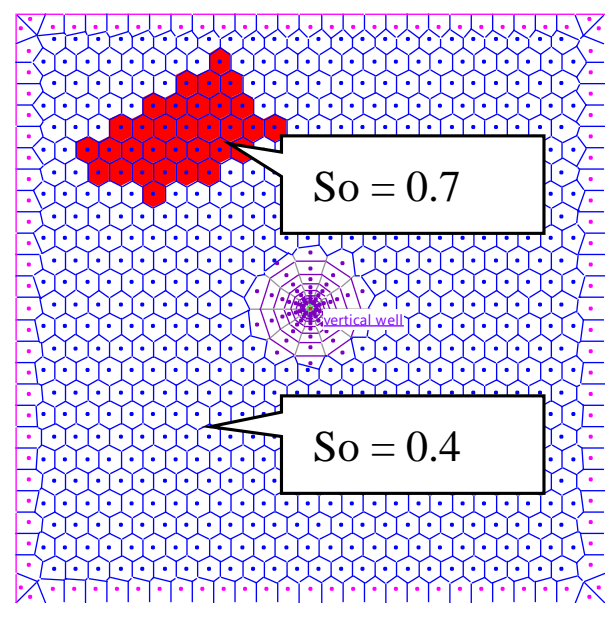

(a) Grid and oil saturation

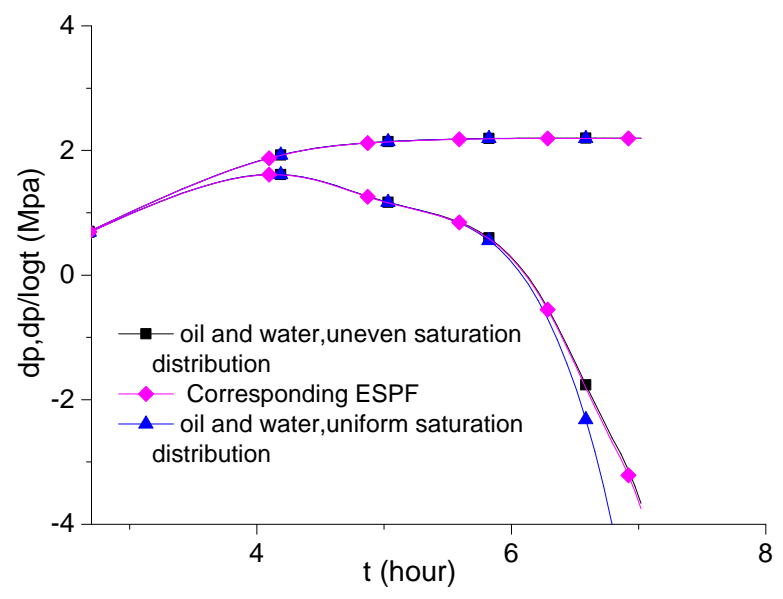

(b) Buildup period

Fig. 2. Region with oil saturation $S_{o}=0.7$ is small and far away from the well.

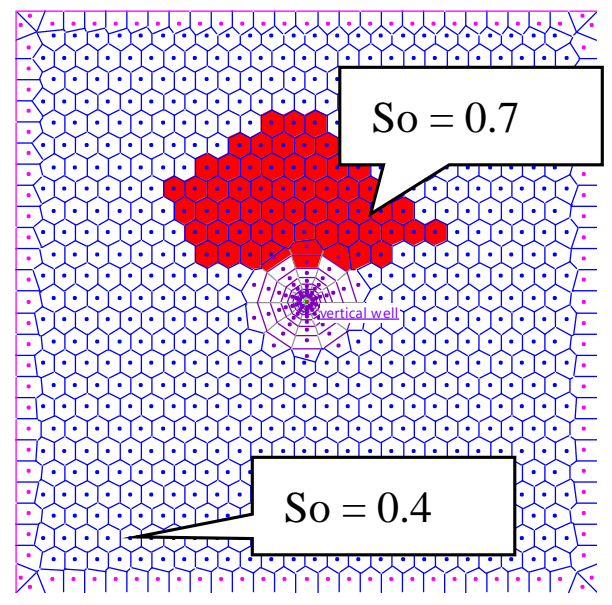

(a) Grid and place where oil saturation is 0.7

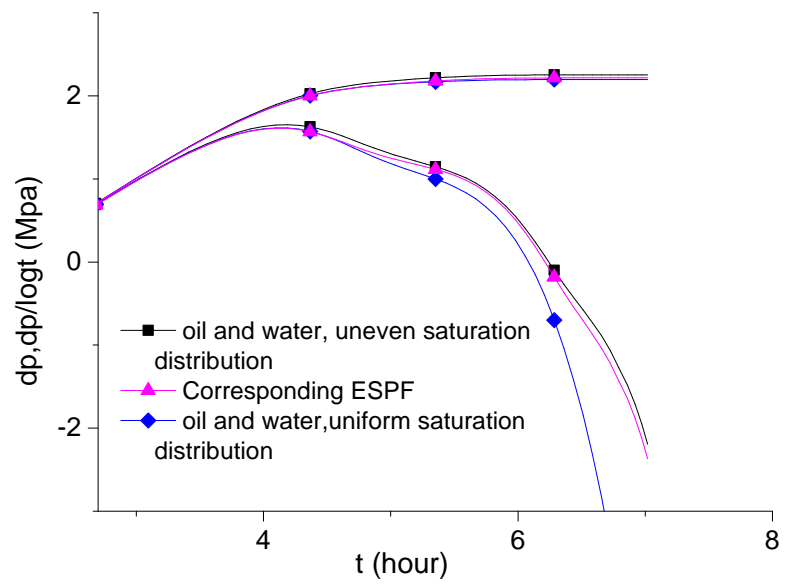

(b) Log-log curves of the buildup period

Fig. 3. Region with oil saturation $S_{o}=0.7$ is much bigger and nearer.

value and thus larger error might be expected.

In comparison to the previous results, the curve of the ESPF in Fig. 3(b) is obviously different from the curve of oil-water two-phase with a uniform saturation distribution. Therefore, in this situation, the ESPF model is sensitive to the value of saturation difference.

\subsection{Experiment 3: Constant pressure boundary con- ditions}

In this experiment, the oil reservoir boundary conditions are set as constant pressure to observe the performance of the ESPF model. Fig. 4 gives saturation distribution and the $\log -\log$ curves in the buildup period.

Fig. 4(b) shows that the equivalent single phase and the two-phase flow have a very good agreement even when the region with oil saturation of 0.7 is so big and the saturation is so sharp. At the same time, the pressure derivative curve of ESPF and two-phase flow with uniform saturation are parallel at the later period, which is different from the case of a closed reservoir.

The reason is related to the saturation variance. In the closed reservoir, both water saturation and water mobility decrease gradually. Therefore, more oil will flow into the well and oil saturation will decrease more quickly as time goes on. In constant pressure boundary conditions, the water flows into the reservoir from the boundary, and the water mobility decreases more slowly and thus oil saturation change slowly compared to the closed boundary condition. This phenomenon is important because in the old oilfield there are so many injection wells that the closed boundary condition is not suitable for a single well, which will improve the effectiveness of the ESPF model.

\subsection{Experiment 4: One production well and four injection wells}

In this model, there are four injection wells and one pro- 


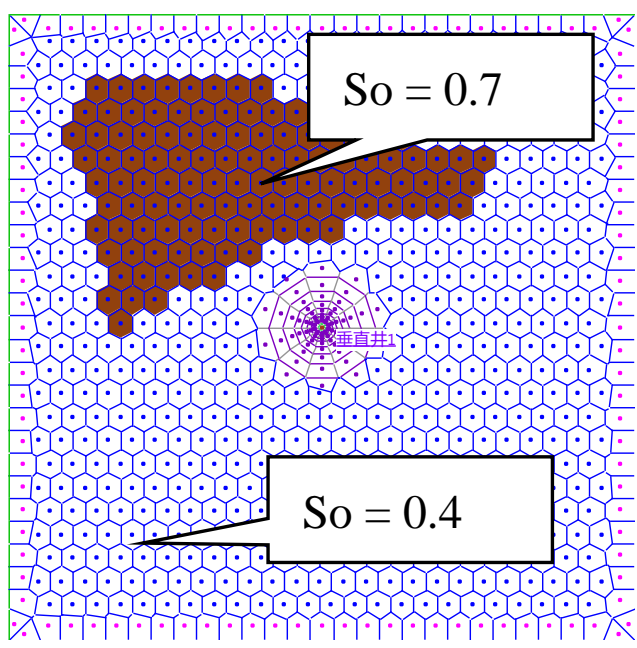

(a) Saturation distribution

Fig. 4. Model with constant pressure boundary condition.

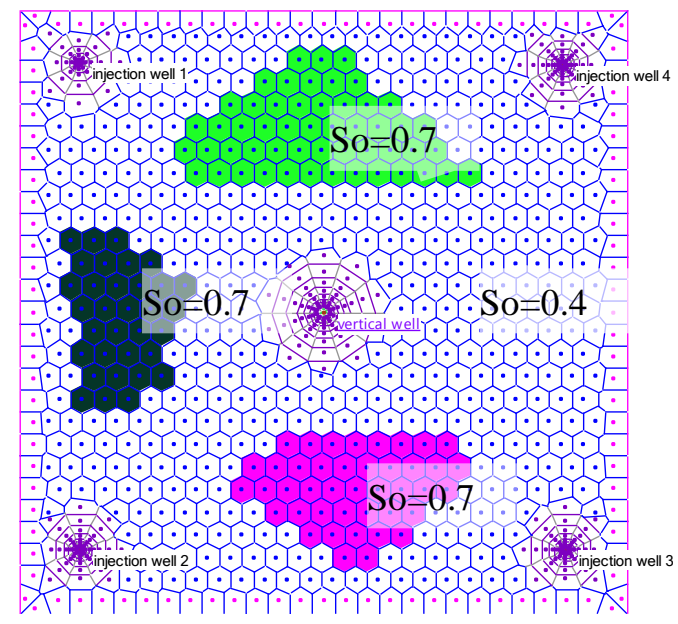

(a) Grid and saturation distribution

Fig. 5. Model with multi-wells and multi saturation distributions.

duction well to simulate the probable remaining oil distribution caused by water flooding as shown in Fig. 5. The oil saturation in one region is 0.7 , while the oil saturation in other place is 0.4 as shown in Fig. 5(a). Fig. 5(b) shows that the equivalent single phase and the two-phase flow also have a very good agreement. The curves of equivalent single phase are different from curves of oil-water two-phase with uniform saturation distribution at the middle part of the curves.

For more practical pressure data interpretation, more than 10 wells are usually added around the measured well to consider the influence of the adjacent wells and to reduce influence of the boundary condition. At this situation, the closed boundary condition is always used.

There are sharp saturation boundaries in Fig. 5(a). and the saturation difference is 0.3 , which is very sharp. A more realistic case that saturation difference is 0.1 is given in Fig. 6(a).

Fig. 6(b) shows that when the saturation difference in

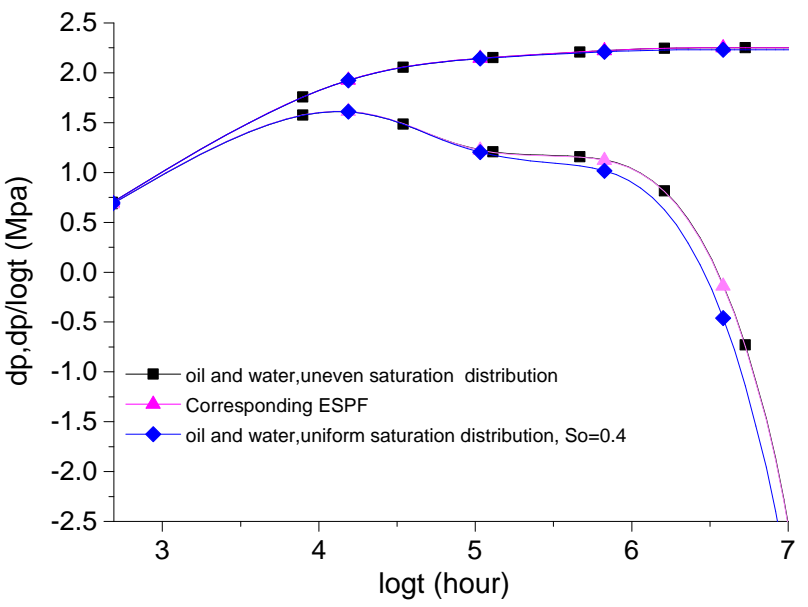

(b) Log-log curves of the buildup period

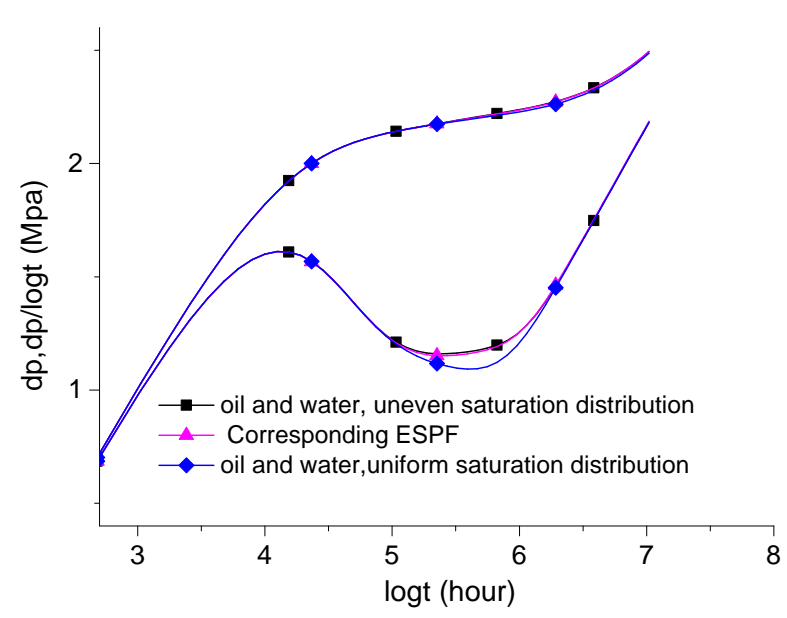

(b) Log-log curves of the buildup period

spatial position becomes small, the equivalent single phase and the two-phase flow have a better agreement and their curves almost coincide. This is because that when the saturation difference is small, saturation change in oil-water two-phase flow is also small, and thus the error caused by the assumption of stationary saturation is small. That is the reason why the equivalent single-phase matches the two-phase flow better when the saturation difference in different regions is small.

This finding is very important because in realistic reservoirs oil saturation varies in space continuously and thus the saturation difference is small. The equivalent single-phase flow model has a better performance at this situation, which means that the equivalent model will be more useful.

Fig. 6(b) also shows that the curves of equivalent single phase and the curves of oil-water two-phase flow with uniform saturation distribution detach at the middle part of the curves. This observation indicates that the small saturation difference influences the log-log curves the same way as the big satura- 


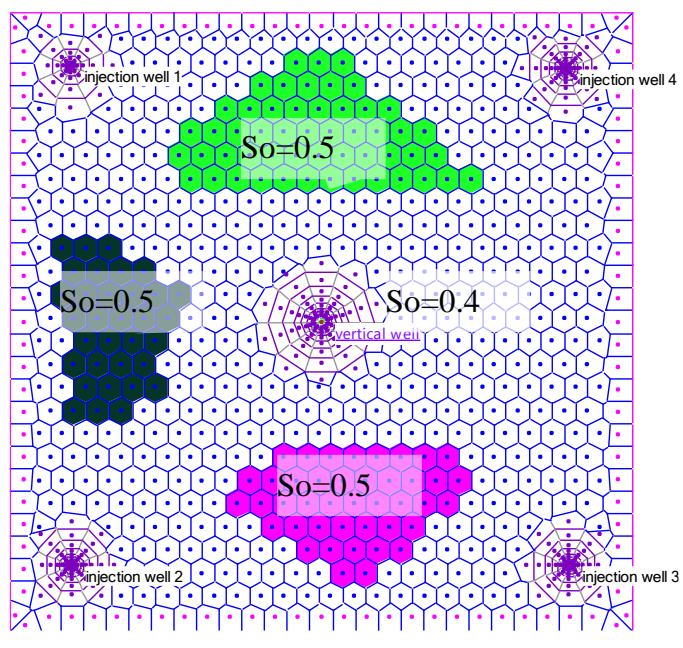

(a) Grid and saturation distribution

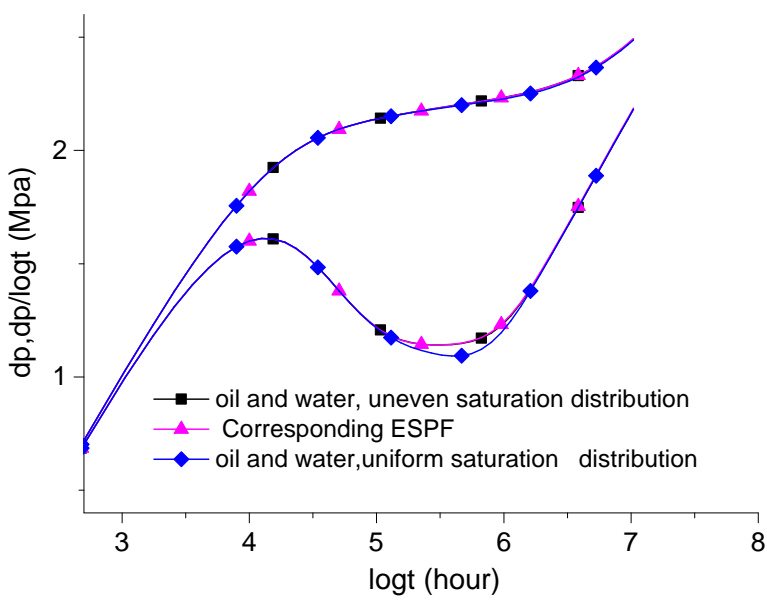

(b) Log-log curves of the buildup period

Fig. 6. Log-log curves of the buildup period.

tion difference.

\section{Discussion}

\subsection{The old reservoir is always oil-water two-phase}

A virgin reservoir may be under sufficient pressure to push hydrocarbons to surface. As the fluids are produced, the pressure will often decline which leads to lower production. In order to maintain the production and pressure of reservoir, water is usually injected back into the reservoir to increase pressure or displace oil from the reservoir, and push it towards a well. At the same time, the reservoir pressure is always maintained above the bubble pressure to avoid gas breakthrough. Therefore, for the old fields, the fluid is always oil-water twophase flow.

The assumption of two-phase flow is a little restrictive. In practice, the delay of water injection schemes may cause local reservoir pressures to fall below the bubble point, leading to the liberation of gas. Moreover, gas caps may exist. However, two-phase flow without gas caps does exist, for example, Daqing oil field in China, which has been developed for more than forty years with high oil output. The observation indicates that the reservoir pressure is maintained above the bubble pressure is also common. Therefore, the equivalent singlephase flow is useful in some oil fields.

\subsection{Justification of the assumption of equivalent single-phase flow}

During the process ofpressure interpretation, simulation time only lasts several months, and thus the distribution of oil saturation does not vary much.

Actually, many fields use analytical well test software to interpret the buildup pressure of oil-water two-phase flow, which is also based on the assumption of the stationary saturation. The composite reservoir model is often used in well test analysis taking into account of the complex geology and the saturation distribution. When the region composite model is used in the homogenous reservoir, the composite model reflects the variation of viscosity decided by the ratio of oil and water saturation, which does not change with time in the analytical well test for sure.

\subsection{Increase the pressure interpretation Speed}

During the process of interpretation of the reservoir parameters using numerical well test based on the pressure data, the grid is so small that the smallest grid may be less than $1 \mathrm{~m}^{2}$, leading to the very small time step and the long compuation time, which is a big burden for users.

The ESPF model can convert the oil-water two-phase flow into single-phase flow. When parameters such as $\mu_{w}, \mu_{o}$, $B_{o}, B_{w}$, relative permeability curves and the phase saturation distribution are given, the parameters $B_{e}, \mu_{e}, B_{a}$ can be calculated from Eqs. (8)-(10). With these parameters, the ESPF model can be used to simulate the oil-water two-phase flow.

Generally, the ESPF model cannot really replace the oilwater two-phase flow under any situation. However, the ESPF model has a good agreement with the oil-water two-phase flow during a short time span, which has been illustrated in above experiments. Given that the ESPF model interprets the pressure data directly or offers good initial values for the true oil-water two-phase pressure data interpretation, the efficiency of the interpretation of oil-water pressure data has been significanlty improved.

\subsection{Multiple relative permeabilities}

In practice, relative permeability function varies in different parts of the field. From the definition of $\mu_{e}$ and $B_{e}$ we know that it is easy for ESPF model to incorporate different relative permeability curves. 


\section{Conclusions}

In this work, we first proposed the ESPF model based on the two-phase flow equations, then discussed application of the model and the corresponding algorithms, finally carried out numerical simulations based on the PEBI grids proving the effectiveness of the ESPF model. Our conclusions could be summarized as below.

1) The ESPF model is based on assumption of the stationary saturation. Therefore, the model is valid if the saturation change is small. The ESPF model can be used to interpret the oil-water two-phase pressure data measured in shutin wells. The saturation can be interpreted from the equivalent viscosity.

2) The larger the saturation difference between adjacent regions, the larger disagreement between the results of the ESPF model and the oil-water two-phase flow for the reservoir with single well and closed boundary conditions. However, the constant pressure boundary condition will reduce the disagreement greatly. The reason is that water phase mobility changes much smaller with the constant pressure boundary condition.

3) That injection wells around the production well reduce the effect of the saturation difference to reduce the error of the ESPF model.

4) The ESPF model offers good initial values for the true oilwater two-phase pressure data interpretation. Thus, it will greatly save the time of numerical well test interpretation for the oil-water two-phase flow.

5) Due to the hydraulically fractured treatment in tight reservoirs, oil-water or water-gas two-phase flow model should be considered, which will be included in our future work.

\section{Nomenclature}

$$
\begin{aligned}
& p=\text { Reservoir pressure, } \mathrm{Pa} \\
& t=\text { Time, } \mathrm{s} \\
& h=\text { Thickness of layer, } \mathrm{m} \\
& C_{r}=\text { Rock compressibility, } \mathrm{Pa}^{-1} \\
& \phi=\text { Porosity } \\
& K=\text { Permeability, } \mathrm{m}^{2} \\
& K_{r}=\text { Relative permeability } \\
& \mu=\text { Viscosity, } \mathrm{Pa} \cdot \mathrm{s} \\
& q=\text { Flow rate, } \mathrm{m}^{3} \cdot \text { day }^{-1} \\
& Q=\text { Surface rate, } \mathrm{m}^{3} \cdot \mathrm{day}^{-1} \\
& C_{l}=\text { Compressibility of } l \text { phase fluid, } \mathrm{Pa}^{-1} \\
& B=\text { Formation volume factor } \\
& B_{a}=\text { Accumulation formation volume factor } \\
& r_{w}=\text { Well Radius, } \mathrm{m} \\
& r_{e}=\text { Equivalent well radius, } \mathrm{m} \\
& C=\text { Well bore storage factors, } \mathrm{m}^{3} \cdot \mathrm{Pa}^{-1} \\
& S=\text { Well bore skin, dimensionless number } \\
& T_{i j}=\text { Conductivity between grid } i \text { and } j, \mathrm{~m}^{-1} \cdot \mathrm{Pa} \cdot \mathrm{s}
\end{aligned}
$$

\section{Subscript}

$i, j=$ Grid $i, j$

$o, w, e=$ Oil phase, water phase, and equivalent single phase

$$
n=\text { Old time }
$$

\section{Superscript}

$r e f=$ Parameter under Reference pressure

\section{Acknowledgments}

This work was supported by grants from National Key Science and Technology Project (2017ZX05009005-002).

Open Access This article is distributed under the terms and conditions of the Creative Commons Attribution (CC BY-NC-ND) license, which permits unrestricted use, distribution, and reproduction in any medium, provided the original work is properly cited.

\section{References}

Alcalde, O.R., Teufel, L.W. Diagnosis of formation damage by rock deformation/compaction through numerical Well-Test simulations. Paper SPE 98053 Present at International Symposium and Exhibition on Formation Damage Control, Lafayette, Louisiana, USA, 15-17 February, 2006.

Ayan, C., Lee, W.J. The effects of multiphase flow on the interpretation of buildup tests. Paper SPE 15537 Presented at the 61st Annual Technical Conference and Exhibition held in New Orleans, LA, 5-8 October, 1986.

Cai, J.C., Hu, X., Xiao, B., et al. Recent developments on fractal-based approaches to nanofluids and nanoparticle aggregation. Int. J. Heat Mass Transf. 2017, 105(10): 623-637.

Chu, W.C., Reynolds, A.C., Raghavan, R. Pressure transient analysis of two-phase flow problems. SPE Form. Eval. 1986, 1(2):151-164.

Earlougher, R.C., Miller, F.G., Mueller, T.D. Pressure buildup behavior in a two-well gas-oil system. Soc. Petrol. Eng. J. 1967, 7(2): 195-204.

Fetkovich, M.J., Vienot, M.E. Rate normalization of buildup pressure by using afterflow data. J. Pet. Technol. 1984, 36(12): 1813-1824.

Li, D.L., Xu, C.Y., Wang, J.Y.L., et al. Effect of Knudsen diffusion and Langmuir adsorption on pressure transient response in shale gas reservoir. J. Pet. Sci. Eng. 2014, 124(10): 146-154.

Li, D.L., Zha, W.S., Liu, S.F., et al. Pressure transient analysis of low permeability reservoir with pseudo threshold pressure gradient. J. Pet. Sci. Eng. 2016a, 147(5): 308316.

Nnadi, M., Onyekonwu, M. Numerical welltest analysis. Paper SPE 88876 Presented at the 28th Annual SPE international technical conference and exhibition in Abuja, Nigertia, 2-4 August, 2004.

Palagi, C.L., Aziz, K. Use of voronoi grid in reservoir simulation. Paper SPE 22889 Presented at the 1991 SPE Annual Technical Conference and Exhibition, Dallas, 6-9 October, 1991.

Perrine, R.L. Analysis of pressure buildup curves. Drill. Prod. Prac. 1956, 482-509. 
Pinzon, C.L., Chen, H.Y., Teufel, L.W. New mexicotech numerical well test analysis of stress-sensitive reservoirs. Paper SPE 71034 Prepared at the SPE Rocky Mountain Petroleum Technology Conference held in Keystone, Colorado, 21-23 May, 2001.

Raghavan, R. Well test analysis: wells producing by solution gas drive wells. Soc. Petrol. Eng. J. 1976, 16(4): 196-208.

Raghavan, R. Well test analysis for multiphase flow. Paper SPE 14098 Presented at the SPE 1986 International Meeting on Petroleum Engineering held in Beijing, China, 17-20 March, 1986.

Raghavan, R. A note on the theoretical foundations for multiphase pressure analysis for flow in porous media. J. Pet. Sci. Eng. 2009, 68(1): 81-88.
Thompson, L.G., Reynolds, A.C. Well testing for radially heterogeneous reservoirs under single and multiphase flow conditions. SPE Form. Eval. 1997, 12(1): 57-64.

Yue, X.A., Wei, H.G. Low pressure gas percolation characteristic in ultra-low pemeability porous media. Transp. Porous Media 2010, 85(1): 333-345.

Zhang, T., Li, Z., Adenutsi, C.D., et al. A new model for calculating permeability of natural fractures in dualporosity reservoir. Adv. Geo-Energy Res. 2017, 1(2): 86-92.

Zhao, Y., Shan, B.C., Zhang, L.H., et al. Seepage flow behaviors of multi-stage fractured horizontal wells in arbitrary shaped shale gas reservoirs. J. Geophys. Eng. 2016, 13(5): 1-10. 